\title{
Everted Pedicled Temporalis Fascial Flap to Augment Pedicled Pericranial Flap for Lax Duraplasty in Decompressive Craniotomy: A Cost-Effective Procedure
}

\author{
Rajesh Bhosle ${ }^{1}$ Shamshuddin Sr Patel ${ }^{1} \quad$ Dimble Raju ${ }^{1} \quad$ Nabanita Ghosh ${ }^{2} \quad$ Prasad Krishnan $^{1, \odot}$ \\ ${ }^{1}$ Department of Neurosurgery, National Neurosciences Centre, \\ Address for correspondence Prasad Krishnan MS, MCh, \\ Calcutta, West Bengal, India \\ ${ }^{2}$ Department of Neuroanesthesiology, National Neurosciences \\ Department of Neurosurgery, National Neurosciences Centre, \\ Centre, Calcutta, West Bengal, India \\ 2nd Floor, Peerless Hospital Campus, 360, Panchasayar, Garia, \\ Kolkata 700094, West Bengal, India \\ (e-mail: prasad.krishnan@rediffmail.com).
}

J Neurosci Rural Pract 2021;12:438-440.
Abstract
Keywords
- decompressive craniotomy
- pericranium
- temporalis fascia
- duraplasty
- pedicled flap
- cranioplasty

Decompressive craniotomy is a commonly performed surgery to relieve raised intracranial pressure. At the end of the procedure, it is the convention to cover the exposed brain by performing a lax duraplasty which allows for both brain expansion and provides protection to the underlying parenchyma. Various commercially available dural substitutes are used for this purpose. These have the drawback of being both expensive and nonvascularized. We propose a technique of using pericranium along with everted temporalis fascia (both being locally harvested vascularized pedicle flaps) that can suffice in a vast majority of cases for covering the brain.

\section{Introduction}

Decompressive craniotomy is commonly performed for relieving raised intracranial pressure. ${ }^{1}$ It entails removal of the skull and durotomy (commonly a stellate durotomy) and, when appropriate, dealing with the offending pathology (contusionectomy, clot evacuation, etc.) as well. Prior to closure of the scalp flap, conventionally, a lax duraplasty is done. This has well documented advantages of decreasing the incidence of CSF leak, ${ }^{2,3}$ preventing infections ${ }^{3}$ and obviating cortical injury and irritation when a subgaleal drain is placed. An added advantage of performing a duraplasty is ease of dissection and avoidance of cortical injury when a second surgery (interval cranioplasty) is performed. ${ }^{3}$ Autologous fascia lata and pericranial grafts were used to provide this cover by suturing it to the durotomy margins, but increasingly

published online April 1, 2021
DOI https://doi.org/

$10.1055 / \mathrm{s}-0041-1726661$ ISSN 0976-3147 commercial available artificial dural substitutes are being used. ${ }^{4}$ We propose a technique of using pedicled pericranial and everted temporalis fascial flaps for duraplasty that is both inexpensive and effective.

\section{Technique}

Initially, the incision is marked and the scalp is infiltrated with local anesthetic and adrenaline solution. In the traditional "trauma flap," we prefer to extend the incision in a curvilinear manner across the midline (if the anticipated flap is very large). This has the advantage of both allowing supply to the scalp flap by the opposite supratrochlear artery as well as enabling us to harvest a larger amount of pericranium for cover. The galea and skin are lifted off and reflected anteroinferiorly taking care not to injure the pericranium. The latter

(c)2021. Association for Helping Neurosurgical Sick People.

This is an open access article published by Thieme under the terms of the Creative Commons Attribution-NonDerivative-NonCommercial-License, permitting copying and reproduction so long as the original work is given appropriate credit. Contents may not be used for commercial purposes, or adapted, remixed, transformed or built upon. (https://creativecommons.org/licenses/by-nc-nd/4.0/).

Thieme Medical and Scientific Publishers Pvt. Ltd. A-12, 2nd Floor, Sector 2, Noida-201301 UP, India 


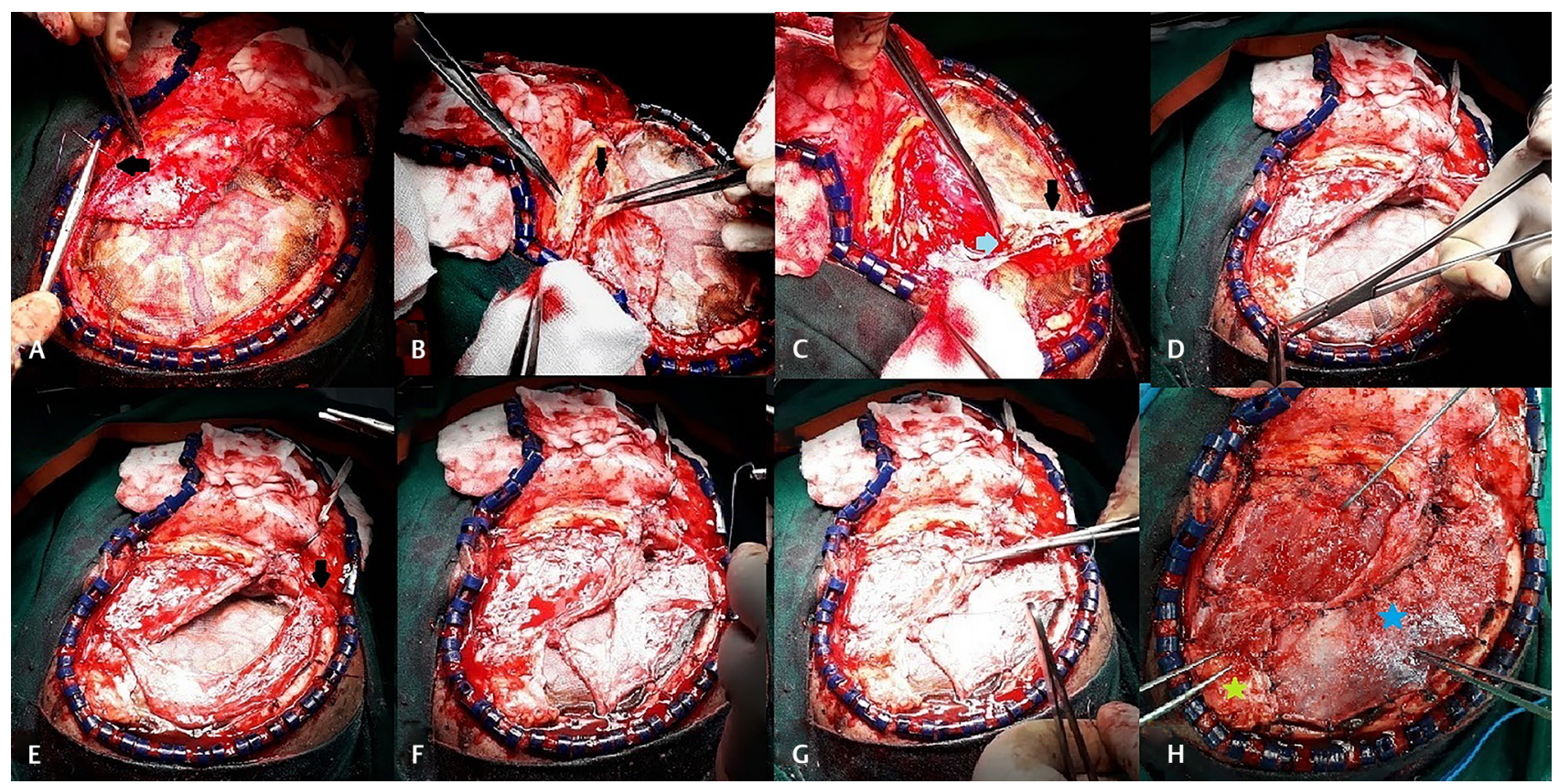

Fig. 1 Clinical photograph showing (A) Surgicel being layered on the brain and temporalis muscle being loosely affixed anteriorly and posteriorly (black arrow); (B) temporalis fascia being incised inferiorly; (C) raised by sharp dissection off the temporalis muscle (black arrow) keeping the posterosuperior attachment intact (blue arrow); (D) sutured to the posterior margins of the craniotomy defect to cover the exposed brain; (E) the pericranium being placed loosely without tension on the brain with site to attachment of the pedicle anteriorly shown (black arrow); $(\mathbf{F}, \mathbf{G})$ pericranium being sutured superiorly to the craniotomy margins and inferiorly to the temporalis muscle; and (H) after complete duraplasty showing the pericranium (blue star), everted temporalis fascia (green star), and temporalis muscle with dissector on it.

is then sharply cut with a knife along the proposed line of craniotomy (superiorly and posteriorly) and inferiorly at its attachment with the temporalis fascia at the superior temporal line and is reflected anteriorly up to the orbital margins, thus retaining it as a pedicled flap for closure. The temporalis muscle is then erased off the bone in the usual manner and reflected inferiorly. Craniotomy and durotomy are then done in the usual manner.

At the time of closure, we prefer to lay a sheet of Surgicel on the exposed brain. The temporalis muscle is re-sutured loosely posteriorly and anteriorly with two or three stitches (-Fig. 1A). The pericranial flap is then brought back to cover the exposed brain above the temporalis muscle. In large craniotomies, the flap often is found to fall short of the posterior margin and even if it does reach the posterior extent is often on stretch, and thus, the purpose of a lax duraplasty may be foiled. In such cases, we then sharply dissect the temporalis fascia off the temporal muscle fibers, keeping its posterosuperior attachment in place and evert it (creating a pedicled everted fascial flap) to provide cover to posterior part of the exposed brain ( - Fig. 1B-D). This is attached to the craniotomy margins superiorly (-Fig. 1E) and posteriorly. The pericranium in turn is loosely sutured to the superior margins of the temporalis to the everted temporalis flap and to the craniotomy margins ( - Fig. 1F-H). Scalp closure is then done in the usual manner over a closed suction drain.

\section{Discussion}

Pericranium, historically, has been the most common material used to perform a duraplasty. It has the advantage of being harvestable from the local site unlike autologous fascia lata grafts that require a separate incision. However, most surgeons harvest a free rather than a pedicled flap since retaining a flap with a pedicle decreases the amount available to cover the exposed brain or even if just sufficient or may necessitate suturing it to the craniotomy margins under tension preventing brain expansion. Larger craniotomies also dissuade surgeons from preserving pericranium as a flap if it is deemed at the start of surgery that the craniotomy defect would be too large to be covered by pericranium alone. Everting the temporalis fascia as a pedicled flap allows us in conjunction with the pedicled pericranial flap to perform a lax duraplasty.

Nonvascularized tissues suffer from the drawback of undergoing slow lysis even as they act as a template for the growth of connective tissue through them. ${ }^{3}$ The rate at which they degrade cannot be predicted and often only a very thin neodura is encountered at the time of cranioplasty, which may be breached. Pedicled flaps allow quicker healing as they possess intrinsic vascular supply. ${ }^{3}$ A study by Morales-Avalos et $\mathrm{al}^{5}$ has compared the proportion of elastic fibers and tissue density of pericranium, dura mater, and temporalis fascial flap and have concluded that the temporalis fascial flap is superior to pericranium as a dural graft. 
The availability of artificial dural substitutes has led to the decreasing use of locally available tissues for providing brain cover. However, they are foreign bodies and, though more robust, they too are avascular and have a small but finite risk of infection and may be immunogenic as well. ${ }^{4}$ Moreover, in resource constrained settings, they impose an unnecessary financial burden on the patient or the hospital.

\section{Conclusion}

Though the temporalis fascia cannot be used to cover large defects, in view of the fact that it is accessible through the same incision, can be retained with a pedicle and can be harvested easily, we feel this may be a useful and cost-effective solution to augment pericranium if the latter falls short or is found to be stretched and under tension at the time of closure.

\section{Conflict of Interest}

None declared.

\section{References}

1 Krishnan P, Chowdhury SR. Posture-dependent aphasia: focal cortical dysfunction in the sinking scalp flap syndrome. J Neurosci Rural Pract 2015;6(2):225-227

2 Giovanni S, Della Pepa GM, La Rocca G, et al. Galea-pericranium dural closure: can we safely avoid sealants? Clin Neurol Neurosurg 2014;123:50-54

3 Velnar T, Gradisnik L. Soft tissue grafts for dural reconstruction after meningioma surgery. Bosn J Basic Med Sci 2019;19(3):297-303

4 Sabatino G, Della Pepa GM, Bianchi F, et al. Autologous dural substitutes: a prospective study. Clin Neurol Neurosurg 2014;116:20-23

5 Morales-Avalos R, Soto-Domínguez A, García-Juárez J, et al. Characterization and morphological comparison of human dura mater, temporalis fascia, and pericranium for the correct selection of an autograft in duraplasty procedures. Surg Radiol Anat 2017;39(1):29-38 